\title{
ON SUPPLEMENT ELEMENTS IN LATTICES
}

\author{
CELIL NEBIYEV
}

Received 10 February, 2019

\begin{abstract}
In this work, some properties of supplement elements in lattices are investigated. Some relation between lying above and (weak) supplement elements also studied. Some properties of supplement submodules in modules which given in [8] are generalized to lattices. Let $a$ be a supplement of $b$ in a lattice $L$. If $a / 0$ has at least one maximal $(\neq a)$ element, then it is possible to define a bijective map between the maximal elements $(\neq a)$ of $a / 0$ and the maximal elements $(\neq 1)$ of $1 / b$. Let $a$ be a supplement element in a lattice $L$. If $L$ is amply supplemented, then $a / 0$ is also amply supplemented. If $L$ is weakly supplemented, then $a / 0$ is also weakly supplemented.
\end{abstract}

2010 Mathematics Subject Classification: 06C05; 06C15

Keywords: lattices, small elements, supplemented lattices, complemented lattices

\section{INTRODUCTION}

Throughout this paper, all lattices are complete modular lattices with the smallest element 0 and the greatest element 1 . Let $L$ be a lattice, $a, b \in L$ and $a \leq b$. A sublattice $\{x \in L \mid a \leq x \leq b\}$ is called a quotient sublattice, denoted by $b / a$. Let $L$ be a lattice, $a, b \in L$ and $a<b$. If there is no element $c$ of $L$ such that $a<c<b$, we say that $a$ is covered by $b$ (or $b$ covers $a$ ) and denoted by $a \prec b$. An element $a$ of a lattice $L$ is called an atom if $0 \prec a$. An element $a^{\prime}$ of a lattice $L$ is called a complement of $a$ if $a \wedge a^{\prime}=0$ and $a \vee a^{\prime}=1$, this case we denote $1=a \oplus a^{\prime}$ ( $a$ and $a^{\prime}$ also is called direct summands of $L$ ). $L$ is called a complemented lattice if each element has at least one complement in $L$. An element $a$ of $L$ is said to be small or superfluous in $L$ and denoted by $a \ll L$ if $b=1$ for every element $b$ of $L$ such that $a \vee b=1$. The meet of all maximal elements $(\neq 1)$ of a lattice $L$ is called the radical of $L$ and denoted by $r(L)$. An element $c$ of $L$ is called a supplement of $b$ in $L$ if it is minimal for $b \vee c=1 . a$ is a supplement of $b$ in a lattice $L$ if and only if $a \vee b=1$ and $a \wedge b \ll a / 0$. A lattice $L$ is said to be supplemented if every element of $L$ has a supplement in $L$. We say that an element $b$ of $L$ lies above an element $a$ of $L$ if $a \leq b$ and $b \ll 1 / a$. An element $a$ of $L$ is called a weak supplement of $b$ in $L$ if $a \vee b=1$ and $a \wedge b \ll L$. A lattice $L$ is said to be weakly supplemented, if every element of $L$ has a weak supplement in $L$. An element $a \in L$ has ample supplements 
in $L$ if for every $b \in L$ with $a \vee b=1, a$ has a supplement $b^{\prime}$ in $L$ with $b^{\prime} \leq b . L$ is called an amply supplemented lattice, if every element of $L$ has ample supplements in $L$. It is clear that every supplemented lattice is weakly supplemented and every amply supplemented lattice is supplemented. An element $c$ of a lattice $L$ is called a generalized supplement or Rad-supplement of $b$ in $L$ if $b \vee c=1$ and $b \wedge c \leq r(c / 0)$. $L$ is said to be generalized supplemented (or Rad-supplemented) if every element of $L$ has a generalized supplement in $L$. It is clear that every supplemented lattice is generalized supplemented, but the converse is not true in general (See [3, Example $1]$ ). Let $L$ be a lattice. It is defined $\beta_{*}$ relation on the elements of $L$ by $a \beta_{*} b$ with $a, b \in L$ if and only if for each $t \in L$ such that $a \vee t=1$ then $b \vee t=1$ and for each $k \in L$ such that $b \vee k=1$ then $a \vee k=1$.

More details about (amply) supplemented lattices are in [1,2,6]. More results about (amply) supplemented modules are in [5,9]. Some important properties of supplement submodules are in [8]. The definition of generalized supplemented lattices and some properties of them are in [3]. The definition of $\beta_{*}$ relation on lattices and some properties of this relation are in [7]. The definition of $\beta^{*}$ relation on modules and some properties of this relation are in [4].

In this paper, it is generalized some properties of supplement submodules and lying above to lattices. It is studied some relationships between (weak) supplement elements and lying above by Proposition 6, Proposition 7, Lemma 14, Lemma 15 and Corollary 6. If $a$ is a supplement of $b$ in a lattice $L$, it is defined a bijective map between maximal $(\neq a)$ elements of $a / 0$ and maximal $(\neq 1)$ elements of $1 / b$ by Proposition 3. It is proved that a lattice $L$ is amply supplemented if and only if every element of $L$ lies above a supplement element in $L$ by Lemma 16.

Lemma 1 (See [6, Lemma 12.3]). In any modular lattice $[(c \vee d) \wedge b] \leq$ $[c \wedge(b \vee d)] \vee[d \wedge(b \vee c)]$ holds for every $b, c, d \in L$.

Lemma 2 (See [6, Lemma 7.4]). Let $L$ be a lattice, $a, b \in L$ and $a \leq b$. If $a \ll b / 0$ then $a \ll L$.

Lemma 3 (See [6, Lemma 7.5]). In a lattice L let $c^{\prime} \ll c / 0$ and $d^{\prime} \ll d / 0$. Then $c^{\prime} \vee d^{\prime} \ll(c \vee d) / 0$.

Lemma 4 (See [6, Lemma 7.6]). If $a \ll L$, then $a \leq r(L)$.

Lemma 5. Let $L$ be a lattice, $a, b \in L$ and $a \leq b$. Then $b$ lies above $a$ if and only if $a \beta_{*} b$.

Proof. $(\Rightarrow)$ See [7, Theorem 3].

$(\Leftarrow)$ Let $b \vee t=1$ with $t \in 1 / a$. Since $a \beta_{*} b, a \vee t=1$ and since $a \leq t, t=1$. Hence $b \ll 1 / a$ and $b$ lies above $a$.

Lemma 6 (See [7, Lemma 2]). Let $L$ be a lattice and $a, b, c \in$ L. If $a \vee b=1$ and $(a \wedge b) \vee c=1$, then $a \vee(b \wedge c)=b \vee(a \wedge c)=1$. 
Lemma 7. Let $L$ be a lattice, $a, b, k \in L$ and $1=a \vee b$. If $a<k<1$, then $0<k \wedge b<b$.

Proof. Since $a<k$ and $1=a \vee b, k=k \wedge 1=k \wedge(a \vee b)=a \vee(k \wedge b)$ and since $k \neq a$, we get $k \wedge b \neq 0$. Since $a \vee(k \wedge b)=k \neq 1$ and $1=a \vee b$, we get $k \wedge b \neq b$. Hence $0<k \wedge b<b$ holds.

Lemma 8 (See [6, Proposition 12.2]). Let a be a supplement of $b$ in L. If $b$ is $a$ maximal $(\neq 1)$ element of $L$, then $a \wedge b$ is the unique maximal $(\neq a)$ element of $a / 0$ and $r(a / 0)=a \wedge b$ holds.

Lemma 9. Let $L$ be a lattice and $a, b \in L-\{1\}$. If $1=a \vee b$ and $b$ is an atom, then $a$ is a maximal $(\neq 1)$ element of $L$.

Proof. If $k \in L$ and $a<k<1$, then by Lemma 7, $0<k \wedge b<b$. Since $b$ is an atom, this is contradiction. Hence $a$ is a maximal $(\neq 1)$ element of $L$.

Corollary 1. Let $a$ be a supplement of $b$ in $L$. Then $b$ is maximal $(\neq 1)$ if and only if $0 \prec a$ or $a \wedge b \prec a$.

Proof. Clear from Lemma 8 and Lemma 9.

\section{SOME PROPERTIES OF SUPPLEMENT ELEMENTS}

Lemma 10. Let a be a supplement of $b$ in $L$ and $c \in a / 0$. Then $c \ll L$ if and only if $c \ll a / 0$.

Proof. $(\Rightarrow)$ Let $c \vee t=a$ with $t \in a / 0$. Then $b \vee c \vee t=b \vee a=1$ and since $c \ll L, b \vee t=1$. By minimality of $a$, we get $t=a$. Hence $c \ll a / 0$.

$(\Leftarrow)$ Clear from Lemma 2 .

Proposition 1. Let a be a supplement element in $L$. If $L$ is weakly supplemented, then a/0 is also weakly supplemented.

Proof. Let $x \in a / 0$. Since $L$ is weakly supplemented, there exists an element $y$ of $L$ such that $x \vee y=1$ and $x \wedge y \ll L$. Here $a=a \wedge 1=a \wedge(x \vee y)=x \vee(a \wedge y)$ holds. Since $x \wedge a \wedge y=x \wedge y \ll L$, by Lemma 10, $x \wedge a \wedge y \ll a / 0$. Hence $a / 0$ is weakly supplemented.

Lemma 11. Let $a$ be a supplement of $b$ in $L$ and $x, y \in a / 0$. Then $y$ is a supplement of $x$ in $a / 0$ if and only if $y$ is a supplement of $b \vee x$ in $L$.

Proof. $(\Rightarrow)$ Let $y$ be a supplement of $x$ in $a / 0$ and $b \vee x \vee z=1$ with $z \leq y$. Because of $x, y \in a / 0$ and $z \leq y, x \vee z \leq a$. Since $a$ is a supplement of $b$ in $L$, $a=x \vee z$. Since $y$ is a supplement of $x$ in $a / 0, z=y$. Hence $y$ is a supplement of $b \vee x$ in $L$.

$(\Leftarrow)$ Let $y$ be a supplement of $b \vee x$ in $L$. So, $b \vee x \vee y=1$ and $(b \vee x) \wedge y \ll y / 0$. Since $x \vee y \leq a$ and $a$ is a supplement of $b$ in $L, x \vee y=a$ and $x \wedge y \leq(b \vee x) \wedge y \ll$ $y / 0$. Hence $y$ is a supplement of $x$ in $a / 0$. 
Corollary 2. Let $a$ and $b$ be mutual supplements in $L, x$ be a supplement of $y$ in $a / 0$ and $z$ be a supplement of $t$ in $b / 0$. Then $x \vee z$ is a supplement of $y \vee t$ in $L$.

Proof. By Lemma 11, $x$ is a supplement of $b \vee y$ and $z$ is a supplement of $a \vee t$ in $L$. Hence $x \wedge(b \vee y) \ll x / 0$ and $z \wedge(a \vee t) \ll z / 0$. By Lemma 1 and Lemma 3, $(x \vee z) \wedge(y \vee t) \leq[x \wedge(y \vee t \vee z)] \vee[z \wedge(x \vee y \vee t)]=[x \wedge(y \vee b)] \vee[z \wedge(a \vee t)] \ll$ $(x \vee z) / 0$ and since $1=a \vee b=x \vee y \vee z \vee t=x \vee z \vee y \vee t, x \vee z$ is a supplement of $y \vee t$ in $L$.

Corollary 3. Let $1=a \oplus b$ in $L, x$ be a supplement of $y$ in $a / 0$ and $z$ be $a$ supplement of $t$ in $b / 0$. Then $x \vee z$ is a supplement of $y \vee t$ in $L$.

Proof. Clear from Corollary 2.

Proposition 2. Let a be a supplement element in $L$. If $L$ is amply supplemented, then a/0 is also amply supplemented.

Proof. Let $a$ be a supplement of $b$ in $L$ and $a=x \vee y$ with $x, y \in a / 0$. Since $1=a \vee b=b \vee x \vee y$ and $L$ is amply supplemented, there exists a supplement $y^{\prime}$ of $b \vee x$ in $L$ with $y^{\prime} \leq y$. By Lemma $11, y^{\prime}$ is a supplement of $x$ in $a / 0$. Hence $a / 0$ is amply supplemented.

Lemma 12. Let $a$ be a supplement of $b$ in $L$ and $x$ be a maximal $(\neq a)$ element of $a / 0$. Then $b \vee x$ is a maximal $(\neq 1)$ element of $L$ and $x=a \wedge(b \vee x)$.

Proof. Since $a$ is a supplement of $b$ in $L, 1=a \vee b$ and $a \wedge b \ll a / 0$. By Lemma 4, we get $a \wedge b \leq r(a / 0) \leq x$. By modularity, $a \wedge(b \vee x)=(a \wedge b) \vee x=x$ holds. Since $x$ is a maximal $(\neq a)$ element of $a / 0$ and $\frac{1}{b \vee x}=\frac{a \vee b}{b \vee x} \cong \frac{a}{a \wedge(b \vee x)}=\frac{a}{x}, b \vee x$ is a maximal $(\neq 1)$ element of $L$.

Lemma 13. Let $L$ be a lattice and $a \in L$. If $k$ is a maximal $(\neq 1)$ element of $L$ and $a \not \leq k$, then $a \wedge k$ is a maximal $(\neq a)$ element of $a / 0$.

Proof. Since $a \not k k$, we get $a \wedge k \neq a$. Let $a \wedge k<t \leq a$ with $t \in a / 0$. Then $t \nless k$ and by maximality of $k, k \vee t=1$. Here $a=a \wedge 1=a \wedge(k \vee t)=(a \wedge k) \vee t=t$. Hence $a \wedge k$ is a maximal $(\neq a)$ element of $a / 0$.

Proposition 3. Let $a$ be a supplement of $b$ in a lattice $L$. If $a / 0$ has at least one maximal $(\neq a)$ element, then it is possible to define a bijective map between the maximal $(\neq a)$ elements of a 0 and maximal $(\neq 1)$ elements of $1 / b$.

Proof. Let $\Gamma=\{k \in a / 0 \mid k$ is maximal $(\neq a)$ in $a / 0\}$ and $\Lambda=\{t \in L \mid b \leq$ $t$ and $t$ is maximal $(\neq 1)$ in $1 / b\}$. We can define a map

$$
f: \Gamma \longrightarrow \Lambda, \quad k \longrightarrow f(k)=b \vee k .
$$

By Lemma $12, b \vee k$ is maximal $(\neq 1)$ in $1 / b$. Hence $f$ is a function. Let $f\left(k_{1}\right)=$ $f\left(k_{2}\right)$ for $k_{1}, k_{2} \in \Gamma$. Then $b \vee k_{1}=b \vee k_{2}$ and $a \wedge\left(b \vee k_{1}\right)=a \wedge\left(b \vee k_{2}\right)$. By 
Lemma $12, k_{1}=a \wedge\left(b \vee k_{1}\right)$ and $k_{2}=a \wedge\left(b \vee k_{2}\right)$. Hence $k_{1}=k_{2}$ and $f$ is injective.

Let $t \in \Lambda$. Then $t$ is a maximal $(\neq 1)$ element of $L$ and $b \leq t$. Since $1=a \vee b$ and $b \leq t$, by maximality of $t, a \not \leq t$. Then by Lemma $13, a \wedge t$ is a maximal $(\neq a)$ element of $a / 0$ and $a \wedge t \in \Gamma$. By definition of $f$ and the modularity of $L, f(a \wedge t)=$ $b \vee(a \wedge t)=(b \vee a) \wedge t=1 \wedge t=t$ and $f$ is surjective.

Hence $f$ is bijective.

The following Proposition can be given as a consequence of [3, Theorem 2]. It is proved by different way as follows.

Proposition 4. Let $a$ be a supplement of $b$ in $L$ and $b \neq 1$. If $r(L) \ll L$, then there exists a maximal $(\neq 1)$ element $t$ of $L$ such that $b \leq t$.

Proof. If $a \leq r(L)$, then $a \ll L$ and since $1=a \vee b, b=1$. This is contradiction. Hence $a \not \leq r(L)$ and there exists a maximal $(\neq 1)$ element $k$ of $L$ such that $a \not \leq k$. By Lemma $13 a \wedge k$ is a maximal $(\neq a)$ element of $a / 0$ and by Lemma $12, t=$ $b \vee(a \wedge k)$ is a maximal $(\neq 1)$ element of $L$ with $b \leq t$.

The following Proposition can be given as a consequence of [3, Lemma 2]. It is proved by different way as follows.

Proposition 5. Let a be a supplement of $b$ in $L$. Then $r(a / 0)=a \wedge r(L)$.

Proof. Assume that $a \leq r(L)$. If $k$ is a maximal $(\neq a)$ element of $a / 0$, by Lemma $12, b \vee k$ is a maximal $(\neq 1)$ element of $L$ and $k=a \wedge(b \vee k)$. Then by $a \leq r(L) \leq$ $b \vee k, a=a \wedge(b \vee k)=k$. This is contradiction. Hence $a / 0$ have no maximal $(\neq a)$ elements and since $a \leq r(L), r(a / 0)=a=a \wedge r(L)$.

Now assume that $a \not \leq r(L)$. Then there exists a maximal $(\neq 1)$ element $t$ of $L$ such that $a \not \leq t$. By Lemma $13, a \wedge t$ is a maximal $(\neq a)$ element of $a / 0$ and hence $a / 0$ has at least one maximal $(\neq a)$ element. By using Lemma 12, we can see that $r(a / 0)=$ $\wedge\{x \mid x$ is maximal $(\neq a)$ in $a / 0\}=\wedge\{a \wedge(b \vee x) \mid x$ is maximal $(\neq a)$ in $a / 0\}=$ $a \wedge[\wedge\{b \vee x \mid x$ is maximal $(\neq a)$ in $a / 0\}]$.

Since $r(L) \leq b \vee x$ for every maximal $(\neq a)$ element $x$ of $a / 0, r(L) \leq \wedge\{b \vee$ $x \mid x$ is maximal $(\neq a)$ in $a / 0\}$ and $a \wedge r(L) \leq a \wedge[\wedge\{b \vee x \mid x$ is maximal $(\neq a)$ in $a / 0\}]=r(a / 0)$. By Lemma 13, for every maximal $(\neq 1)$ element $t$ of $L, a \wedge$ $t=a$ or $a \wedge t$ is a maximal $(\neq a)$ element of $a / 0$. Then by using the definition of $r(L)$ we can see that $r(a / 0) \leq \wedge\{a \wedge t \mid t$ is maximal $(\neq 1)$ in $L\}=a \wedge[\wedge\{t \mid$ $t$ is maximal $(\neq 1)$ in $L\}]=a \wedge r(L)$.

Hence $r(a / 0)=a \wedge r(L)$.

Proposition 6. Let $L$ be a lattice, $a, b \in L, a \leq b$ and $b$ lies above $a$ in $L$. If $a$ and $b$ have weak supplements in $L$, then they have the same weak supplements in $L$.

Proof. Since $b$ lies above $a$, by Lemma $5, a \beta_{*} b$. Then by [7, Theorem 4], the desired is obtained. 
Proposition 7. Let $L$ be a lattice, $a, b \in L, a \leq b$ and $b$ lies above $a$. If $a$ and $b$ have supplements in $L$, then they have the same supplements in $L$.

Proof. Since $b$ lies above $a$, by Lemma 5, $a \beta_{*} b$. Then by [7, Theorem 4], the desired is obtained.

Lemma 14. Let a be a weak supplement of $b$ in $L$ and $k \leq a$. Then $k$ is a weak supplement of $b$ in $L$ if and only if a lies above $k$ in $L$.

Proof. $(\Rightarrow)$ Since $k$ is a weak supplement of $b$ in $L, 1=k \vee b$ and $k \wedge b \ll L$. By modularity, $a=a \wedge 1=a \wedge(k \vee b)=k \vee(a \wedge b)$. Let $a \vee t=1$ with $t \in 1 / k$. Then $1=a \vee t=k \vee(a \wedge b) \vee t=(a \wedge b) \vee t$ and since $a \wedge b \ll L, t=1$. Hence $a \ll 1 / k$ and $a$ lies above $k$ in $L$.

$(\Leftarrow)$ By Lemma 5, $k \beta_{*} a$ and since $a \vee b=1, k \vee b=1$. In addition to this, $k \wedge b \leq a \wedge b \ll L$. Hence $k$ is a weak supplement of $b$ in $L$.

Lemma 15. Let a be a weak supplement of $b$ in $L$ and $b \leq c$. Then $a$ is a weak supplement of $c$ in $L$ if and only if $c$ lies above $b$ in $L$.

Proof. $(\Rightarrow)$ Since $a$ is a weak supplement of $c$ in $L, c$ is a weak supplement of $a$ in $L$. Then by Lemma $14, c$ lies above $b$ in $L$.

$(\Leftarrow)$ Since $c$ lies above $b$ in $L$, by Lemma $5, b \beta_{*} c$. Since $a \vee b=1$ and $b \beta_{*} c$, $a \vee c=1$. Let $(a \wedge c) \vee t=1$ with $t \in L$. By Lemma 6, $c \vee(a \wedge t)=1$ and since $b \beta_{*} c, b \vee(a \wedge t)=1$. By using Lemma 6 again, we can see that $(a \wedge b) \vee t=1$ and since $a \wedge b \ll L, t=1$. Hence $a$ is a weak supplement of $c$ in $L$.

Corollary 4. Let $L$ be a weakly supplemented lattice, $a, b \in L$ and $a \leq b$. Then $a$ and $b$ have the same weak supplements in $L$ if and only if $b$ lies above $a$ in $L$.

Proof. Clear from Proposition 6 and Lemma 15.

Corollary 5. Let a be a supplement of $b$ in $L$ and $k \leq b$. Then $a$ is a supplement of $k$ in $L$ if and only if b lies above $k$ in $L$.

Proof. Clear from Lemma 14.

Corollary 6. Let a be a weak supplement of $b$ in L. Then $a$ is a supplement of $b$ in $L$ if and only if a does not lie above any element $c$ with $c<a$.

Proof. Clear from Lemma 14.

Definition 1. Let $L$ be a lattice. $L$ is said to be $\oplus$-supplemented if every element of $L$ has a supplement that is a direct summand of $L$.

Proposition 8. Let $L$ be a lattice. If every element of $L$ has a weak supplement that is a direct summand in $L$, then $L$ is $\oplus$-supplemented.

Proof. Assume that $a$ has a weak supplement $x$ that is a direct summand in $L$ and $1=x \oplus y$ with $y \in L$. Then $x$ is a supplement of $y$ in $L$ and by Corollary $6, x$ does not lie above any element $c$ with $c<x$. Also by Corollary $6, x$ is a supplement of $a$ in $L$. This means that $L$ is $\oplus$-supplemented. 
Proposition 9. A lattice $L$ is weakly supplemented if and only if every element of $L$ lies above a weak supplement in $L$.

Proof. $(\Rightarrow)$ Since $L$ is weakly supplemented, every element of $L$ is a weak supplement in $L$. Since every element of $L$ lies above itself, every element of $L$ lies above a weak supplement in $L$.

$(\Leftarrow)$ Let $a \in L$. By hypothesis, $a$ lies above a weak supplement $b$ in $L$. Let $b$ be a weak supplement of $c$ in $L$. Then $c$ is a weak supplement of $b$ in $L$ and since $a$ lies above $b$ in $L$, by Lemma 15, $c$ is a weak supplement of $a$ in $L$. Hence $L$ is weakly supplemented.

Lemma 16. A lattice $L$ is amply supplemented if and only if every element of $L$ lies above a supplement element in $L$.

Proof. $(\Rightarrow)$ Since $L$ is amply supplemented, then $L$ is supplemented. Let $a \in L$ and $b$ is a supplement of $a$ in $L$. Since $1=a \vee b$ and $L$ is amply supplemented, $b$ has a supplement $x$ in $L$ with $x \leq a$. Since $b$ is a supplement of $a$ in $L, a$ is a weak supplement of $b$ in $L$. Since $x$ is a supplement of $b$ in $L, x$ is a weak supplement of $b$ in $L$. Then by Lemma 14, $a$ lies above $x$ in $L$. Hence every element of $L$ lies above a supplement element in $L$.

$(\Leftarrow)$ Let $a, b \in L$ and $a \vee b=1$. Since every element of $L$ lies above a supplement element in $L, a \wedge b$ lies above a supplement element $x$ in $L$. Let $x$ be a supplement of $y$ in $L$. Then $y$ is a weak supplement of $x$ in $L$ and since $a \wedge b$ lies above $x$ in $L$, by Lemma 15, $y$ is a weak supplement of $a \wedge b$ in $L$. Here $1=(a \wedge b) \vee y$ and $a \wedge b \wedge y \ll L$. By modularity, $b=b \wedge 1=b \wedge((a \wedge b) \vee y)=(a \wedge b) \vee(b \wedge y)$. Then $1=a \vee b=a \vee(a \wedge b) \vee(b \wedge y)=a \vee(b \wedge y)$ and since $a \wedge b \wedge y \ll L, b \wedge y$ is a weak supplement of $a$ in $L$. By hypothesis, there exists a supplement element $b^{\prime}$ in $L$ such that $b \wedge y$ lies above $b^{\prime}$ in $L$. By Lemma $14, b^{\prime}$ is a weak supplement of $a$ in $L$. Here $1=a \vee b^{\prime}$ and $a \wedge b^{\prime} \ll L$. Since $b^{\prime}$ is a supplement in $L$ and $a \wedge b^{\prime} \ll L$, by Lemma $10, a \wedge b^{\prime} \ll b^{\prime} / 0$. Thus $b^{\prime}$ is a supplement of $a$ in $L$. Note that $b^{\prime} \leq b$. Hence $L$ is amply supplemented.

Proposition 10. Let $L$ be a lattice. The following statements are equivalent.

(i) Every element of $L$ lies above a direct summand in $L$.

(i i ) $L$ is amply supplemented and every supplement element in $L$ is a direct summand of $L$.

(i i i ) For every elements $a$ and $b$ of $L$ with $1=a \vee b$, there exists a supplement $x$ of $a$ in $L$ such that $x \leq b$ and $x$ is a direct summand of $L$.

Proof. ( $i) \Rightarrow$ (ii) Since every direct summand of $L$ is a supplement in $L$, by Lemma $16, L$ is amply supplemented. Let $a$ be a supplement element in $L$. By hypothesis, $a$ lies above a direct summand $x$ of $L$. Since $a$ is a supplement element in $L$, by Corollary 6, $a=x$. Hence every supplement element in $L$ is a direct summand of $L$. 
(ii) $\Rightarrow$ (i i i ) Clear.

(iii) $\Rightarrow$ (i) Clearly we can see that $L$ is supplemented. Let $a \in L$ and $b$ is a supplement of $a$ in $L$. By hypothesis, there exists a supplement $x$ of $b$ in $L$ such that $x \leq a$ and $x$ is a direct summand of $L$. Since $b$ is a supplement of $a$ in $L, a$ is a weak supplement of $b$ in $L$. Since $x$ is a supplement of $b$ in $L, x$ is a weak supplement of $b$ in $L$. Then by Lemma 14, $a$ lies above $x$ in $L$. Hence every element of $L$ lies above a direct summand in $L$.

Lemma 17. Let $L$ be a lattice. Then the following statements are equivalent.

(i) $L$ is complemented.

(ii) Every element of $L$ is a direct summand of $L$.

(i i i ) Every element of $L$ is a supplement in $L$.

Proof. (i) $\Leftrightarrow$ (ii) Clear.

(ii ) $\Rightarrow$ (i i i ) Clear, since every direct summand of $L$ is a supplement in $L$.

(iii) $\Rightarrow($ ii $)$ Let $a$ be any element of $L$. By hypothesis $a$ is a supplement in $L$ and there exists an element $b$ of $L$ such that $a$ is a supplement of $b$ in $L$. Here $1=a \vee b$ and $a \wedge b \ll b / 0$. By hypothesis, $a \wedge b$ is a supplement in $L$. Let $a \wedge b$ be a supplement of $k$ in $L$. Then $(a \wedge b) \vee k=1$ and since $a \wedge b \ll L, k=1$. Therefore $a \wedge b$ is a supplement of 1 in $L$. Thus $a \wedge b=0$ and $1=a \oplus b$. Hence every element of $L$ is a direct summand of $L$.

Proposition 11. Let $L$ be a weakly supplemented lattice. Then every weak supplement in $L$ is a supplement in $L$ if and only if $L$ is complemented.

Proof. $(\Rightarrow)$ Let $a \in L$. By hypothesis, $a$ has a weak supplement $b$ in $L$. Then $a$ is a weak supplement of $b$ in $L$. By hypothesis, $a$ is a supplement element in $L$. Hence every element of $L$ is a supplement in $L$ and by Lemma 17, $L$ is complemented.

$(\Leftarrow)$ Since $L$ is complemented, by Lemma 17, every element of $L$ is a supplement in $L$. Hence every weak supplement in $L$ is a supplement in $L$.

\section{REFERENCES}

[1] R. Alizade and E. Toksoy, "Cofinitely weak supplemented lattices," Indian Journal of Pure and Applied Mathematics, vol. 40:5, pp. 337-346, 2009.

[2] R. Alizade and S. E. Toksoy, "Cofinitely supplemented modular lattices," Arabian Journal for Science and Engineering, vol. 36, no. 6, p. 919, 2011, doi: 10.1007/s13369-011-0095-z. [Online]. Available: https://doi.org/10.1007/s13369-011-0095-z

[3] Ç. Biçer, C. Nebiyev, and A. Pancar, "Generalized supplemented lattices," Miskolc Mathematical Notes, vol. 19, no. 1, pp. 141-147, 2018, doi: 10.18514/MMN.2018.1974. [Online]. Available: https://doi.org/10.18514/MMN.2018.1974

[4] G. F. Birkenmeier, F. T. Mutlu, C. Nebiyev, N. Sökmez, and A. Tercan, "Goldie*supplemented modules," Glasgow Mathematical Journal, vol. 52A, pp. 41-52, 2010, doi: 10.1017/S0017089510000212. [Online]. Available: https://doi.org/10.1017/S0017089510000212

[5] J. Clark, C. Lomp, N. Vanaja, and R. Wisbauer, Lifting Modules: Supplements and Projectivity in Module Theory (Frontiers in Mathematics), 2006th ed. Basel: Birkhäuser, 82006.

[6] G. Cǎlugăreanu, Lattice Concepts of Module Theory. Kluwer Academic Publisher, 2000. 
[7] C. Nebiyev and H. H. Ökten, " $\beta$ * relation on lattices," Miskolc Mathematical Notes, vol. 18, no. 2, 2017, doi: 10.18514/MMN.2017.1782. [Online]. Available: https://doi.org/10.18514/MMN.2017. 1782

[8] C. Nebiyev and A. Pancar, "On supplement submodules," Ukrainian Mathematical Journal, vol. 65, no. 7, pp. 1071-1078, 2013, doi: 10.1007/s11253-013-0842-2. [Online]. Available: https://doi.org/10.1007/s11253-013-0842-2

[9] R. Wisbauer, Foundations of Module and Ring Theory. Philadelphia: Gordon and Breach, 1991.

\section{Author's address}

\section{Celil Nebiyev}

Ondokuz Mayıs University, Department of Mathematics, Kurupelit, Atakum, 55270 Samsun, Turkey

E-mail address: cnebiyev@omu.edu.tr 